\title{
Arbeitsklima in der Wirtschaftsinformatik spürbar verschieden
}

Untersuchungen zu Berufen der Wirtschaftsinformatik bleiben weiterhin interessant, wenn sie helfen können, dem mittlerweile länger anhaltenden IT-Fachkräftemangel entgegenzuwirken. Eine Untersuchung der Hochschule Hannover zu Wirtschaftsinformatikern/-informatikerinnen in den ersten zehn Jahren im Beruf zeigt deren berufliche Ziele und die berufliche Zufriedenheit, die sie erlangen. Deutlich wird, dass Frauen und Männer das Arbeitsklima und die Arbeitsbedingungen sehr unterschiedlich wahrnehmen und daher auch unterschiedlich zufrieden sind. Dabei bemängeln Frauen vor allem Merkmale, die mit „fehlender Fairness" zu beschreiben sind.

\section{Georg Disterer}


Der mittlerweile lange anhaltende IT-Fachkräftemangel [1] erfordert weiterhin Untersuchungen des Berufslebens von Wirtschaftsinformatikern/-informatikerinnen. Auf der Basis von Kenntnis und Verständnis zum Berufsleben können die Arbeitsbedingungen gegebenenfalls attraktiver gestaltet werden, um mehr Interessierte auf das Berufsfeld der Wirtschaftsinformatik aufmerksam zu machen.

Dabei stellt der geringe Anteil von Frauen in der Wirtschaftsinformatik grundsätzlich eine Möglichkeit dar, um mehr Interessierte für die Wirtschaftsinformatik zu gewinnen, denn aktuell liegt der Frauenanteil im Studium der Wirtschaftsinformatik bei $21 \%$ gegenüber zum Beispiel $48 \%$ in Fächern der Wirtschaftswissenschaften [2]. Damit stellen sich die Fragen: Welche Merkmale lassen das Berufsfeld der Wirtschaftsinformatik attraktiv erscheinen - und welche Merkmale nicht? Entfalten diese Merkmale gleiche Wirkung auf Frauen wie auf Männer? Im Rahmen der Untersuchung der Hochschule Hannover stehen Merkmale des Berufsfelds, der Berufsbedingungen und des Berufsklimas („occupational climate“) im Mittelpunkt der Diskussion, während etwa andere Untersuchungen typische Merkmale von Arbeitgebern untersuchen (zum Beispiel „organizational climate“ bei [3]).

Für IT-Berufe birgt der geringe Anteil von Frauen auch das Risiko, das von Frauen angebotene Potenzial nicht hinreichend auszuschöpfen und nicht von Diversität und Vielfalt zu profitieren [4-6]. Zudem ist es als Ungerechtigkeit anzusehen, dass interessante und attraktive Berufe der IT-Branche durch soziale und kulturelle Barrieren nicht allen gleichberechtigt offenstehen [4, 7]. Somit sprechen wirtschaftliche und ethische Gründe dafür, Gleichberechtigung und Diversität im Beruf zu schaffen bzw. zu sichern [7, 8].

Und dann ist auch zu berücksichtigen, dass Erwartungen und Ansprüche jüngerer Menschen an ihr Berufsleben heute vermutlich anders aussehen als früher. Trotz einer hohen Leistungsbereitschaft spielen Merkmale wie Vereinbarkeit von Beruf und Familie sowie Ausgewogenheit der Work-LifeBalance eine größere Rolle als früher $[9,10]$.

Daher ist es das Ziel der Untersuchung, Eindrücke und Erfahrungen aus dem Berufsleben von jüngeren Wirtschaftsinformatikern/-informatikerinnen zu erheben und auszuwerten. Dabei wird gefragt, ob Einschränkungen der Zufriedenheit im Beruf Möglichkeiten der Verbesserungen erkennen lassen, um so zur Verringerung des Fachkräftemangels beizutragen. Also zum Beispiel: Nehmen Frauen die Berufsbedingungen und das Klima „anders“ wahr? Sind sie weniger zufrieden? Damit soll ein besseres Verständnis von

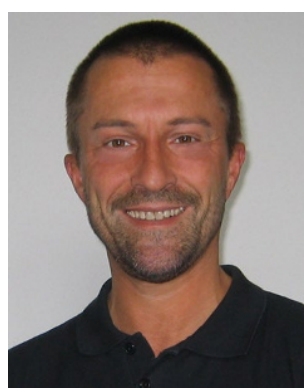

Prof. Dr. Georg Disterer $(\bowtie)$ ist Professor für Wirtschaftsinformatik an der Hochschule Hannover. georg.disterer@hs-hannover.de

Hochschule Hannover, Hannover, Deutschland

Wirtschaftsinformatikern/-informatikerinnen in den ersten Berufsjahren erlangt werden. In diesen Jahren des Eintritts ins Berufsleben werden viele prägende Eindrücke und Erfahrungen aufgenommen und die Arbeitsbedingungen und das Arbeitsklima vermutlich besonders aufmerksam wahrgenommen.

\section{Vorgehensweise}

Um die „berufliche Wirklichkeit“ von Wirtschaftsinformatikern/-informatikerinnen zu erfassen, wurden Absolventen und Absolventinnen des Studiengangs Wirtschaftsinformatik der Hochschule Hannover strukturiert befragt. Zugleich wurden Absolventen und Absolventinnen des Studiengangs Betriebswirtschaftslehre befragt, um damit Vergleichsmöglichkeiten zu schaffen und gegebenenfalls typische Merkmale von Berufen der Wirtschaftsinformatik und deren Wirkungen auf die Beschäftigten identifizieren zu können.

An der Hochschule Hannover haben in den Jahren 2009 bis (August) 2019 insgesamt 1881 Studierende erfolgreich ein einschlägiges Bachelorstudium abgeschlossen, davon 629 ein Studium der Wirtschaftsinformatik und 1250 ein Studium der Betriebswirtschaftslehre. Davon lagen zu 1555 Absolventen und Absolventinnen eine Mail-Adresse vor oder konnte ermittelt werden. Diese Absolventen und Absolventinnen wurden angeschrieben und zur Teilnahme an der Befragung eingeladen. Die Antworten wurden über eine elektronische Plattform im Web anonym und unter Wahrung des Datenschutzes gesammelt. Im Zeitraum April bis Mai 2020 gingen 
insgesamt 424 auswertbare Fragebogen ein; der damit erzielte Rücklauf von 27 \% kann als gut angesehen werden. Damit lagen Antworten von 184 Absolventen und Absolventinnen der Wirtschaftsinformatik und von 240 Absolventen und Absolventinnen der Betriebswirtschaftslehre vor und enthielten Aussagen zu Eindrücken und Erfahrungen ihrer ersten zehn Berufsjahre nach dem Studienabschluss. Nach den Merkmalen Abschlussjahr und Geschlecht waren keine Verzerrungen im Vergleich zur Grundgesamtheit erkennbar. Absolventen und Absolventinnen der Wirtschaftsinformatik waren bei den insgesamt 424 auswertbaren Fragebogen (leicht) überrepräsentiert, was jedoch bei Vergleichen zwischen den Teilkohorten unkritisch ist. Im Folgenden liegt der Fokus der Auswertungen auf den Absolventen und Absolventinnen der Wirtschaftsinformatik, die Abschlüsse zur Betriebswirtschaftslehre wurden zum Vergleich herangezogen.

\section{Ziele im Berufsleben}

Einen wichtigen Ausgangspunkt zur Erfassung der Zufriedenheit von Berufstätigen in der Wirtschaftsinformatik bilden die Ziele, die mit der Berufstätigkeit verfolgt werden. Diese Ziele geben die individuellen Ansprüche an die berufliche Tätigkeit wieder. Mit der Frage „Welche Ziele verfolgen Sie im Berufsleben?" wurden 17 Teilziele zur Auswahl angeboten [11-13], deren Bedeutung oder Stellenwert jeweils mit fünfstufigen, verbal mit „1 sehr unwichtig“ bis „5 sehr wichtig“ verankerten Skalen zu bewerten war.
Aus der Auswahl von 17 Teilzielen wurden die folgenden sieben von den Absolventen und Absolventinnen der Wirtschaftsinformatik nicht als eines der wichtigsten zehn Teilziele ihrer Berufstätigkeit angesehen:

- gut verdienen

- gute Aufstiegs- und Entwicklungsmöglichkeiten haben

- Möglichkeiten zur Übernahme von Koordinations-/Leitungsfunktionen haben

- Möglichkeiten zur beruflichen Weiterbildung/-qualifizierung haben

- jeweiligen Arbeitsumfang bewältigen können

- eigene Ideen entwickeln und umsetzen können

- viel mit Menschen zu tun haben

Erkennbar wird schon hier, dass eine berufliche Karriere von Wirtschaftsinformatikern/-informatikerinnen nicht bedingungslos angestrebt wird $[9,10,13]$.

\section{Top 10 der wichtigsten Berufsziele}

Im Folgenden werden die wichtigsten Berufsziele der Befragten analysiert. In Abb. 1 sind auf der linken Seite die zehn wichtigsten Berufsziele der befragten Männer aufgeführt, in der Reihenfolge der Stellenwerte, wie sie sich aus den Mittelwerten der Angaben der Männer ergeben. Diese Reihenfolge der Berufsziele von Männern spiegelt damit auch die vorherrschenden Werte eines seit vielen Jahren von Männern dominierten Berufsfelds wider [14, 15].

Auf der rechten Seite der Abb. 1 sind die zehn wichtigsten Berufsziele von Frauen aufgeführt, in der Reihenfolge der

Abb. 1 Ziele im Berufsleben von Männern und Frauen (nach Stellenwert, absteigend) $(n=184)$, Skala: „1 sehr unwichtiges Ziel“ bis „5 sehr wichtiges Ziel“" *: Durchschnittl. Bewertung signifikant unterschiedlich zwischen Frauen und Männern $(p<0,01)$

\begin{tabular}{|c|c|c|c|c|}
\hline Ziele im Berufsleben bei Männern & $\begin{array}{l}\text { Rang und } \\
\text { Stellenwert } \\
\text { bei Männern }\end{array}$ & $\begin{array}{l}\text { Unterschiede } \\
\text { bei Rängen }\end{array}$ & $\begin{array}{l}\text { Rang und } \\
\text { Stellenwert } \\
\text { bei Frauen }\end{array}$ & Ziele im Berufsleben bei Frauen \\
\hline respektvollen Umgang erleben & $1(4,6)$ & & $1(4,7)$ & respektvollen Umgang erleben \\
\hline interessante Tätigkeiten ausüben & $2(4,6)$ & & $2(4,6)^{\star}$ & Möglichkeiten zum selbstständigen Arbeiten haben \\
\hline flexible Arbeitszeiten haben & $3(4,5)$ & & $3(4,6)^{*}$ & Anerkennung/Wertschätzung im Beruf erhalten \\
\hline gute Vereinbarkeit von Berufs- $u$. Privatleben erleben & $4(4,5)$ & & $4(4,6)^{*}$ & einen sicheren Arbeitsplatz haben \\
\hline vertrauensvolle Zus.arbeit mit Kollegen/innen erleben & $5(4,4)$ & & $5(4,6)^{\star}$ & Fairness bei Bewertungen/Beurteilungen erleben \\
\hline Möglichkeiten zum selbstständigen Arbeiten haben & $6(4,4)$ & & $6(4,6)^{*}$ & keine Benachteiligung/Diskriminierung erleben \\
\hline Anerkennung/Wertschätzung im Beruf erhalten & $7(4,3)$ & & $7(4,5)$ & gute Vereinbarkeit von Berufs- $u$. Privatleben erleben \\
\hline Fairness bei Bewertungen/Beurteilungen erleben & $8(4,3)$ & & $8(4,5)$ & vertrauensvolle Zus.arbeit mit Kollegen/innen erleben \\
\hline einen sicheren Arbeitsplatz haben & $9(4,2)$ & & $9(4,4)$ & interessante Tätigkeiten ausüben \\
\hline keine Benachteiligung/Diskriminierung erleben & $10(4,2)$ & & $10(4,4)$ & flexible Arbeitszeiten haben \\
\hline
\end{tabular}


Stellenwerte, wie sie sich aus den Mittelwerten der Angaben der Frauen ergeben. Die zehn wichtigsten Berufsziele von Frauen sind identisch mit denen von Männern, allerdings ist die Reihenfolge wesentlich anders. Aus der Gegenüberstellung der Rangfolgen nach Stellenwert wird deutlich, dass bei den Frauen fünf Ziele auf den vorderen Plätzen 2 bis 6 stehen, die bei den Männern lediglich auf den Plätzen 5 bis 10 landen. Die Mittelwerte der Bewertungen dieser fünf Ziele unterscheiden sich dabei zwischen Frauen und Männern signifikant $(p<0,01)$; daher sind sie in der Abbildung mit einem Stern gekennzeichnet.

Also ist bei Männern und Frauen, die in der Wirtschaftsinformatik arbeiten, der Katalog der zehn bedeutendsten Ziele im Berufsleben identisch, die Reihenfolge nach Stellenwert unterscheidet sich allerdings wesentlich.

Eine Faktorenanalyse der Angaben zum Stellenwert einzelner Ziele zeigt, dass vier Faktoren sinnvolle Erklärung liefern, nämlich „Perspektiven“, „Arbeitsinhalte“, „Arbeitsbedingungen“ und „Arbeitsklima“ [12]. Die fünf Ziele, denen von Frauen ein viel höherer Stellenwert zugemessen wird als von Männern (siehe Abb. 1), sind diesen Faktoren wie folgt zuzurechnen:

- Arbeitsinhalte: „Möglichkeiten zum selbstständigen Arbeiten haben"

- Arbeitsbedingungen: „einen sicheren Arbeitsplatz haben“

- Arbeitsklima: „Anerkennung/Wertschätzung im Beruf erhalten“, „Fairness bei Bewertungen/Beurteilungen erleben“ und „keine Benachteiligung/Diskriminierung erleben“

Demnach spielen diese drei Faktoren eine entscheidende Rolle bei den Unterschieden zwischen den Berufszielen von Frauen und Männern, besonders Merkmale des Arbeitsklimas haben bei Frauen einen deutlich höheren Stellenwert als bei Männern.

Bei Absolventen und Absolventinnen der Betriebswirtschaftslehre unterscheiden sich die Ziele von Frauen und Männern weniger deutlich. Wie bei der Wirtschaftsinformatik wird den Zielen „Anerkennung und Wertschätzung im Beruf“, „Fairness bei Bewertungen/Beurteilungen“ und „keine Benachteiligung/Diskriminierung“ von Frauen ein höherer Stellenwert beigemessen als von Männern.

\section{"Geld verdienen" nicht als hohes Ziel genannt}

Zwei Teilergebnisse mögen auffallen: Das zur Auswahl angebotene Ziel der Berufstätigkeit „Geld verdienen“ ist bei Absolventen und Absolventinnen der Wirtschaftsinformatik als auch der Betriebswirtschaftslehre, bei Männern wie bei Frau-

\section{Zusammenfassung}

- Eine Befragung von Wirtschaftsinformatikern/-informatikerinnen in den ersten zehn Berufsjahren zeigt deren wichtigsten beruflichen Ziele sowie die berufliche Zufriedenheit, die sie erlangen.

- Dabei unterscheiden sich die Ziele und die Zufriedenheit recht deutlich zwischen Frauen und Männern.

- Merkmale des Arbeitsklimas verursachen bei Frauen deutlich mehr Einschränkungen der Zufriedenheit als bei Männern.

en, in der Bedeutung nicht unter den Top 10 der Ziele eingereiht. Das deckt sich mit Ergebnissen anderer Untersuchungen [13], auch zu anderen Fachrichtungen [12]. Damit sind Verdienstmöglichkeiten eher als Hygienefaktoren anzusehen, die nur bei mangelnder Erfüllung Unzufriedenheit erzeugen. Dies mag auch beeinflusst sein von der recht positiven Wirtschaftslage der vergangenen Jahre in Deutschland, die Absolventen und Absolventinnen der Wirtschaftsinformatik und Betriebswirtschaftslehre grundsätzlich recht gute Berufs- und Verdienstmöglichkeiten bieten.

\section{"Respekt" als Ziel mit höchstem Wert}

Ebenso auffallend: Das Berufsziel „Respektvollen Umgang erleben" ist bei allen Befragten der Wirtschaftsinformatik und Betriebswirtschaftslehre, bei Männern wie bei Frauen, als das Ziel für das Berufsleben mit dem höchsten Stellenwert benannt und zeugt davon, dass diese Eigenschaft der Unternehmenskultur und des Arbeitsklimas von höchster Bedeutung ist.

\section{Erreichen der beruflichen Ziele}

Als Ergänzung zur Bedeutung und zum Stellenwert der Berufsziele wurde ermittelt, in welchem Ausmaß diese Ziele von Wirtschaftsinformatikern/-informatikerinnen aktuell erreicht werden. Ein Vergleich der Ergebnisse für Männer und Frauen ist in Abb. 2 wiedergegeben. Im oberen Teil ist für Männer den Bedeutungen der einzelnen Ziele die aktuelle Erreichung dieser Ziele gegenübergestellt, nach der Bedeutung der Ziele für Männer gereiht. Im unteren Teil werden diese Angaben für Frauen aufgeführt, nach der Bedeutung der Ziele für Frauen gereiht. 
Augenscheinlich klafft bei Frauen zwischen Bedeutung der Ziele und deren aktuellem Erreichen eine größere Lücke als bei Männern (im unteren Teil von Abb. 2). Diese Lücke ist zu erklären mit den unterschiedlichen Bedeutungen der Ziele für Frauen und Männer. Für Frauen hochrangige Ziele haben bei Frauen eine relativ geringe Zielerreichung, für Männer hochrangige Ziele haben bei Männern eine gute Zielerreichung. Aufgrund dieser Konstellation ist (auch) für die berufliche Zufriedenheit von Frauen ein geringerer Wert als für Männer zu erwarten.

\section{Benachteiligung/Diskriminierung}

Besondere Aufmerksamkeit haben die Aussagen zur Benachteiligung bzw. Diskriminierung verdient. Das Ziel „keine Benachteiligung erleben" hat für Frauen auf einer fünfstufigen Skala mit einem Mittelwert von 4,6 eine signifikant höhere Bedeutung als für Männer mit 4,2 $(p<0,01)$. Das Errei- chen dieses Ziels wird von Frauen im Mittel mit einem Wert von 4,0 eingeschätzt und damit mit einem signifikant geringeren Wert als von Männern (4,3). Das heißt, das Ziel „keine Benachteiligung erleben" ist für Frauen in geringerem Maß erreicht als für Männer. Diese Angaben bestätigen die Ergebnisse anderer Untersuchungen [16].

\section{Zufriedenheit im Beruf}

Zufriedenheit im Beruf gilt heute als wichtiges Kriterium, um letztlich beruflichen Erfolg zu bemessen oder - aus Arbeitgebersicht - als wichtiger Faktor, um Leistung und Einsatzbereitschaft hervorzurufen und zu fördern. Hier wurde die Gesamtzufriedenheit im Beruf erfasst mit der Frage „Wie zufrieden sind Sie insgesamt mit Ihrem Berufsleben?“, mit Antworten auf einer verbal mit „1 sehr zufrieden“ bis „5 sehr unzufrieden" verankerten Skala. Die Erhebung der Berufszufriedenheit mit einer einzigen Frage erscheint etabliert und

\section{Abb. 2 Ziele im Berufsleben und deren Zielerreichung $(n=184)$. Kursiv: signifikant unter- schiedliche Werte bei Frauen und Männern $(p<0,01)$}

Top-10-Ziele der Männer und deren Zielerreichung

1 respektvollen Umgang erleben

2 interessante Tätigkeiten ausüben

3 flexible Arbeitszeiten haben

4 gute Vereinbarkeit von Berufs- $u$. Privatleben erleben

5 vertrauensvolle Zus.arbeit mit Kollegen/innen erleben

6 Möglichkeiten zum selbstständigen Arbeiten haben

7 Anerkennung und Wertschätzung im Beruf erhalten

8 Fairness bei Bewertungen u. Beurteilungen erleben

9 einen sicheren Arbeitsplatz haben

10 keine Benachteiligung oder Diskriminierung erleben

Top-10-Ziele der Frauen und deren Zielerreichung

1 respektvollen Umgang erleben

2 Möglichkeiten zum selbstständigen Arbeiten haben

3 Anerkennung und Wertschätzung im Beruf erhalten

4 einen sicheren Arbeitsplatz haben

5 Fairness bei Bewertungen u. Beurteilungen erleben

6 keine Benachteiligung oder Diskriminierung erleben

7 gute Vereinbarkeit von Berufs- $u$. Privatleben erleben

8 vertrauensvolle Zus.arbeit mit Kollegen/innen erleben

9 interessante Tätigkeiten ausüben

10 flexible Arbeitszeiten haben

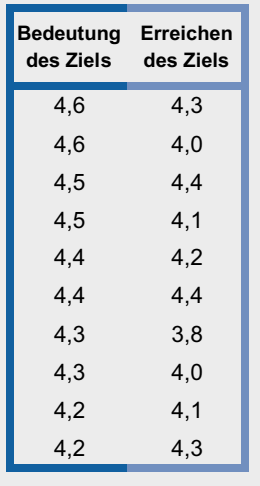

\begin{tabular}{|cc|}
\hline $\begin{array}{c}\text { Bedeutung } \\
\text { des Ziels }\end{array}$ & $\begin{array}{c}\text { Erreichen } \\
\text { des Ziels }\end{array}$ \\
\hline 4,7 & 4,1 \\
$\mathbf{4 , 6}$ & $\mathbf{4 , 2}$ \\
$\mathbf{4 , 6}$ & 3,8 \\
$\mathbf{4 , 6}$ & 4,1 \\
$\mathbf{4 , 6}$ & 3,9 \\
$\mathbf{4 , 6}$ & 4,0 \\
4,5 & 4,1 \\
4,5 & 4,1 \\
4,4 & 3,8 \\
4,4 & 4,4 \\
\hline
\end{tabular}

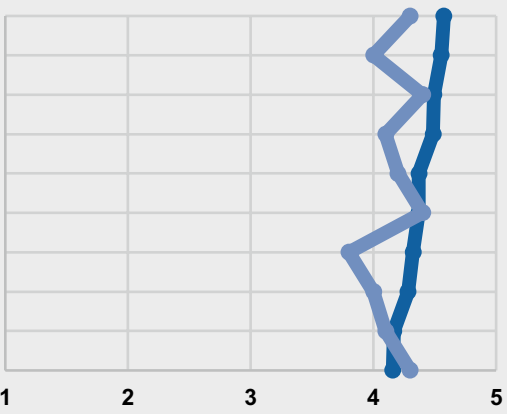

niedrige Bedeutung niedrige Zielerreichung

hohe Bedeutung he Zielerreichung

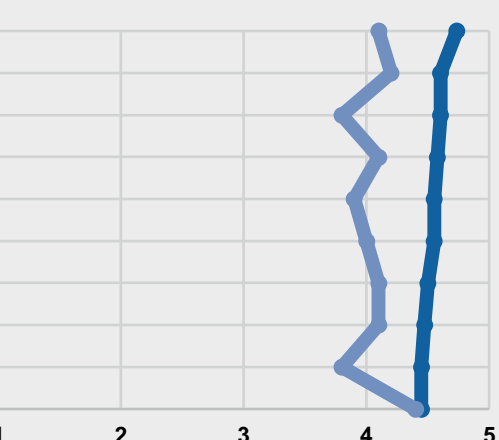

Bedeutung des Ziels

Erreichen des Ziels 
gut begründet $[11,17]$. Ergänzend wurden Dimensionen der Zufriedenheit wie Perspektiven, Arbeitsinhalte, Arbeitsbedingungen, Arbeitsklima sowie Benachteiligung/Diskriminierung erhoben. Diese Dimensionen passen zu jenen Faktoren, die bezüglich der Berufsziele (siehe oben) Ergebnis einer Faktorenanalyse sind. Abb. 3 zeigt die Auswertungen.

Für die Berufszufriedenheit insgesamt ergibt sich auf der fünfstufigen Skala („1 sehr zufrieden“ bis „,5 sehr unzufrieden") eine recht hohe Zufriedenheit, der Mittelwert liegt bei allen Befragten bei 1,9. Bei Männern liegt der Wert bei 1,9, bei Frauen bei 2,1; die Unterschiede zwischen Männern und Frauen sind signifikant $(p<0,01)$. Oder anders formuliert: Der Anteil der befragten Absolventen und Absolventinnen der Wirtschaftsinformatik, die im Berufsleben insgesamt ,unzufrieden“ oder „sehr unzufrieden“ sind, liegt unter $1 \%$. Die damit für Absolventen und Absolventinnen der Wirtschaftsinformatik und der Betriebswirtschaftslehre der Hochschule Hannover erhobenen Werte für die Zufriedenheit im Beruf sind im Vergleich zu den Ergebnissen anderer Untersuchungen deutlich besser [12]. Allerdings muss generell als Limitation von Befragungen zur Berufszufriedenheit beachtet werden, dass eine Verzerrung zugunsten positiver Einschätzungen zu vermuten ist, da (sehr) zufriedene Absolventen und Absolventinnen bereitwilliger freiwillig Auskunft geben werden als nicht so zufriedene.

Die Gesamtzufriedenheit wird durch die Mittelwerte auf den Dimensionen Perspektiven, Arbeitsinhalte, Arbeitsbedingungen, Arbeitsklima sowie Benachteiligung/Diskriminierung gut erklärt. Schon bei den Berufszielen (siehe oben)

\section{Kernthesen}

- Die wichtigen Ziele im Berufsleben sind für Frauen und Männer identisch, allerdings haben diese Ziele einen deutlich verschiedenen Stellenwert bei Frauen und Männern.

- Das Arbeitsklima im Berufsleben hat für Frauen einen höheren Stellenwert als für Männer.

- Frauen nehmen das Arbeitsklima kritischer wahr als Männer: weniger fair, weniger kollegial.

wurde festgestellt, dass Unterschiede zwischen Frauen und Männern den Dimensionen Arbeitsklima und Benachteiligung/Diskriminierung zuzurechnen sind. Entsprechend unterscheiden sich bezüglich dieser beiden Dimensionen die Werte zur Zufriedenheit im Beruf signifikant zwischen Frauen und Männern.

Demnach werden das Arbeitsklima und die wahrzunehmende Benachteiligung/Diskriminierung von Frauen und Männern sehr unterschiedlich wahrgenommen, Frauen spüren hier deutlich mehr Einschränkungen ihrer Zufriedenheit als Männer.

Das ist insoweit typisch für das Berufsfeld der Wirtschaftsinformatik, für das Berufsfeld der Betriebswirtschaftslehre gilt diese Beobachtung nicht, dort sind kaum Unterschiede zwischen den Einschätzungen von Männern und Frauen zu verzeichnen. Derartige Hinweise gibt es schon länger, dass in IT-Berufen das Arbeitsklima von Frauen anders - kriti-

\section{Abb. 3 Zufriedenheit im Berufsleben $(n=176)$ *: Signifikant unterschiedliche Werte bei} Frauen und Männern $(p<0,01)$

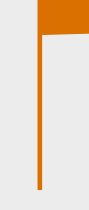

\section{Zufriedenheit mit Berufsleben insgesamt 1,9}

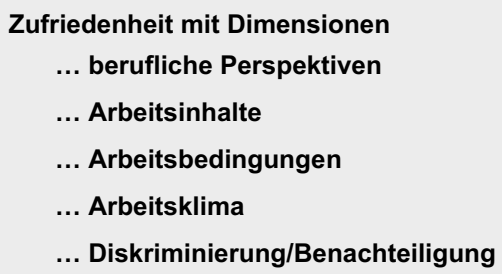

2,6

2,1

2,0

2,2 *

2,1 *

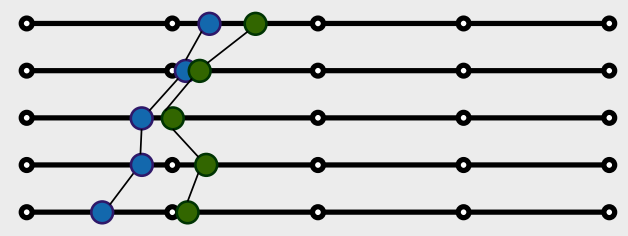




\section{Handlungsempfehlung}

- Maßnahmen zur Verbesserung der Arbeitsatmosphäre können helfen, die berufliche Zufriedenheit von Frauen in Berufen der Wirtschaftsinformatik zu steigern.

- Maßnahmen zur Steigerung des - im weitesten Sinn fairen Umgangs sind am vielversprechendsten.

scher - wahrgenommen wird als von Männern: weniger fair, weniger kollegial [18]. In Abb. 4 sind einzelne Antworten zu den „Einschränkungen des Arbeitsklimas“ wiedergegeben. Erkennbar ist, dass Frauen recht deutliche Einschränkungen wahrnehmen und damit die Atmosphäre im Beruf kritischer erleben. Die in Abb. 4 in der Liste führenden vier Werte sind alle der fairen Behandlung und dem fairen Umgang zuzuschreiben. Also umschreibt der Begriff „Fairness“ eine wichtige Eigenschaft des Arbeitsklimas, zu dem Frauen die Atmosphäre deutlich kritischer wahrnehmen als Männer. Dies ist von Bedeutung, da der wahrgenommenen Fairness ein maßgeblicher Einfluss auf Arbeitszufriedenheit und Einsatzbereitschaft zuzurechnen ist [19].

\section{Diskussion}

Bei anhaltender Diskussion um den Fachkräftemangel in der IT bleibt das Interesse groß an Detailkenntnissen zur Zufriedenheit von Wirtschaftsinformatikern/-informatikerinnen in den ersten Berufsjahren nach Studienabschluss. Die Auswer- tungen einer Befragung von Wirtschaftsinformatikern/-informatikerinnen der Hochschule Hannover geben Hinweise auf einige recht deutliche Unterschiede zwischen den Einstellungen und Wahrnehmungen von Frauen und Männern. Diese Unterschiede stehen wohl einer Erhöhung des Anteils von Frauen im Berufsfeld der Wirtschaftsinformatik im Weg. Der Katalog der beruflichen Ziele von Frauen ist deutlich unterschiedlich zu dem Katalog von Männern, und „ausgerechnet“ die Ziele mit hohem Stellenwert werden von Frauen nicht besonders gut erreicht. Insgesamt wird das Arbeitsklima von Frauen kritischer wahrgenommen als von Männern: weniger fair, weniger kollegial.

In Unternehmen sind bereits einige Maßnahmen etabliert, die Frauen unterstützen, um mit dem vorherrschenden Klima besser zurechtzukommen. Zum Beispiel wird bei Trainings direkt das Missvergnügen aufgegriffen, dass Männer oft ihre Interessen erfolgreicher durchsetzen können, bei der Arbeitsverteilung, bei Planungen oder beim Durchsetzen von Freiräumen. Da Frauen dies oftmals schwerfällt, werden entsprechende Kurse angeboten, in denen trainiert wird, Ausstrahlung, Verhandlungskompetenz und Überzeugungskraft weiterzuentwickeln, um eigene Ansprüche und Wünsche besser durchzusetzen. Maßnahmen dieser Art erscheinen im Grundsatz sinnvoll, da damit Fähigkeiten und Fertigkeiten entwickelt werden, die im Berufsleben wichtig erscheinen. Ob die dazu existierende Ratgeberliteratur zum selbstbewussten Auftreten und mit Tipps und Tricks für eine Karriere im männerdominierten System helfen kann, sei dahingestellt.

\section{Abb. 4 Wahrgenommene Einschränkungen bezüglich des Arbeitsklimas. *: Signifikant unterschiedliche Mittelwerte zwischen Männern und Frauen $(p<0,05)$}

Einschränkungen bezüglich des Arbeitsklimas

im Vergleich mit anderen: nicht gerechte Bezahlung unpassende/ungerechte/unverständliche Stellenbesetzungen u.ä.

wenig Unterstützung und Hilfsbereitschaft untereinander wenig Fairness bei Bewertungen und Beurteilungen wenig vertrauensvolle Zusammenarbeit mit Kollegen/innen wenig Feedback zu Arbeitsleistungen wenig respektvoller Umgang unbefriedigender Umgang zw. Kollegen/innen u. Vorgesetzten wenig offener Umgang mit Fehlern und Problemen wenig Transparenz von Entscheidungen/Plänen des Managements

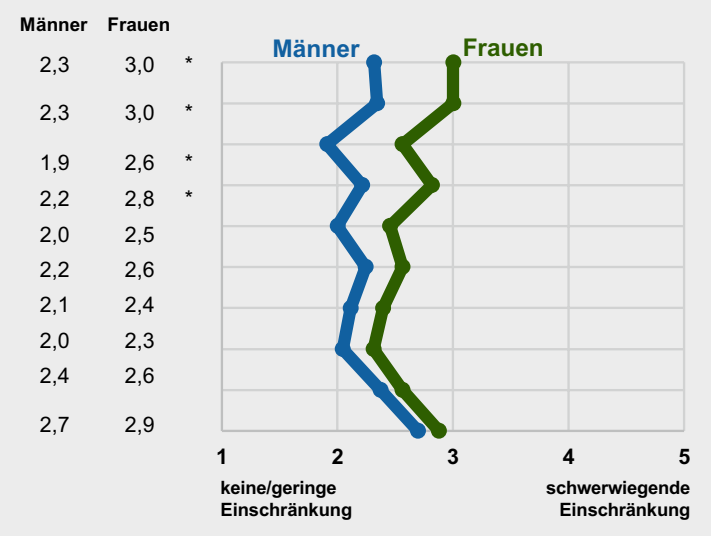


Jedoch bergen solche Maßnahmen die Gefahr, dass Frauen damit eine Assimilation an das vorherrschende Klima angeraten wird, also dass sie etwa ,wie Männer" kommunizieren sollten, um gehört zu werden $[18,20]$. Dies kann aber durchaus anmaßend erscheinen, da die vorherrschende Kultur damit als gegeben angesehen und "gesetzt" wird. Zudem werden damit mögliche Vorteile von Diversität und Pluralität aufs Spiel gesetzt, wenn Frauen ihre unterschiedliche, „andere“ Wahrnehmung der Zusammenarbeit und des Handelns zurückstellen, um nach den Regeln des männlich geprägten Systems zu agieren - dieses System bliebe damit unberührt und unverändert.

Daher erscheinen Maßnahmen zum Teambuilding und zur Personalentwicklung geeigneter, die tatsächliche Werte und Normen der Zusammenarbeit aufgreifen, gegebenenfalls infrage stellen und für alle so ändern, dass die berufliche Zufriedenheit bei Frauen und Männern noch größer wird.

Funding. Open Access funding enabled and organized by Projekt DEAL.

Open Access Dieser Artikel wird unter der Creative Commons Namensnennung 4.0 International Lizenz veröffentlicht, welche die Nutzung, Vervielfältigung, Bearbeitung, Verbreitung und Wiedergabe in jeglichem Medium und Format erlaubt, sofern Sie den/die ursprünglichen Autor(en) und die Quelle ordnungsgemäß nennen, einen Link zur Creative Commons Lizenz beifügen und angeben, ob Änderungen vorgenommen wurden.

Die in diesem Artikel enthaltenen Bilder und sonstiges Drittmaterial unterliegen ebenfalls der genannten Creative Commons Lizenz, sofern sich aus der Abbildungslegende nichts anderes ergibt. Sofern das betreffende Material nicht unter der genannten Creative Commons Lizenz steht und die betreffende Handlung nicht nach gesetzlichen Vorschriften erlaubt ist, ist für die oben aufgeführten Weiterverwendungen des Materials die Einwilligung des jeweiligen Rechteinhabers einzuholen.

Weitere Details zur Lizenz entnehmen Sie bitte der Lizenzinformation auf http://creativecommons.org/licenses/by/4.0/ deed.de.

\section{Literatur}

[1] CEDEFOP (2016). Fachkräftemangel und-überschuss in Europa. Thessaloniki: Europäisches Zentrum für die Förderung der Berufsbildung CEDEFOP.
[2] Statistisches Bundesamt (2020). Bildung und Kultur, Studierende an Hochschulen SS 2019, 11(4.1). www.destatis.de/DE/Themen/ Gesellschaft-Umwelt/Bildung-Forschung-Kultur/Hochschulen/ Publikationen/Downloads-Hochschulen/studierende-hochschulen-ss-2110410197314.pdf. Zugegriffen: 5. Juli 2020.

[3] Oehlhorn, C. E., Maier, C., Laumer, S., \& Weitzel, T. (2019). Attracting young IT professionals: an empirical study using the theory of attractive quality. In Proc. Conference on computers and people research conference SIGMIS-CPR, ACM (S. 85-93).

[4] Cerf, V., \& Johnson, M. (2016). Enrollments Explode! But diversity students are leaving. Communications of the ACM, 59(4), 7.

[5] LeRouge, C. M., Wiley, J. W., \& Maertz, C. P. (2013). A comparison of job satisfaction between IT and non-IT women incumbents in clerical, professional, and managerial positions. Database for Advances in Information Systems, 44(2), 39-54.

[6] Fatourou, P., Papageorgiou, Y., \& Petousi, V. (2019). Women are needed in STEM: European policies and incentives. Communications of the ACM, 62(4), 52-57.

[7] Barton, D., Devillard, S., \& Hazlewood, J. (2015). Gender equality: taking stock of where we are. McKinsey Quarterly, 4, 86-89.

[8] Oehlhorn, C., Laumer, S., \& Maier, C. (2017). About wellconsidered decisions, favorable alternatives and sudden ideas: a qualitative research to identify beliefs that influence women to study information systems in Germany. In Proc. der 13. Int. Tagung Wirtschaftsinformatik (WI 2017) (S. 365-379).

[9] Urbach, N., \& Ahlemann, F. (2016). IT-Management im Zeitalter der Digitalisierung - Auf dem Weg zur IT-Organisation der Zukunft. Berlin: Springer Gabler.

[10] Schuth, M., Brosi, P., \& Welpe, I. M. (2018). Recruiting Women in IT: A Conjoint-Analysis Approach. In Proc. of the 51th Hawaii Int. Conference on System Sciences (HICSS) (S. 5096-5105).

[11] Pietrzyk, I., \& Graser, A. (2017). Gütekriterien des Fragebogens des Kooperationsprojekts Absolventenstudien. Working Paper \# 6. Kassel: Int. Centre for Higher Education Research Kassel INCHER. [12] Fabian, G., Hillmann, J., Trennt, F., \& Briedis, K. (2016). Hochschulabschlüsse nach Bologna. Hannover: Deutsches Zentrum für Hochschul- und Wissenschaftsforschung DZHW.

[13] Gärtig-Daugs, A., Förtsch, S., \& Schmid, U. (2014). Alumnae Tracking - Frauenkarrieren in der Informatik. FifF-Kommunikation, 3, 30-36.

[14] Kirton, G., \& Robertson, M. (2018). Sustaining and advancing IT careers: women's experiences in a UK-based IT company. Journal of Strategic Information Systems, 27(2), 157-169.

[15] Förtsch, S., Gärtig-Daugs, A., Buchholz, S., \& Schmid, U. (2018). Keep it going girl! An empirical analysis of gender differences and inequalities in computer sciences. Journal of Gender, Science and Technology, 10(2), 265-286.

[16] Funk, C., \& Parker, K. (2018). Women and men in STEM often at odds over workplace equity. Washington: Pew Research Center. 
[17] Wanous, J. P., Reichers, A. E., \& Hudy, M. J. (1997). Overall job satisfaction: how good are single-item measures? Journal of Applied Psychology, 82(2), 247-252.

[18] Gunter, R., \& Stambach, A. (2005). Differences in men and women scientist's perceptions of workplace climate. Journal of Women and Minorities in Science and Engineering, 11(1), 97-116.

[19] Brooks, N. G., Riemenschneider, C. K., \& Armstrong, D. J. (2019). The information technology professional: a two-wave study of factors impacting commitment and satisfaction. Communications of the Association for Information Systems, 44, 600-629.

[20] Bronstein, P., \& Farnsworth, L. (1998). Gender differences in faculty experiences of interpersonal climate and processes for advancement. Research in Higher Education, 39, 557-585. 